\title{
Fibrosarcomatous changes and expression of CD34+ and apolipoprotein-D in dermatofibrosarcoma protuberans
}

\author{
Emanuela Palmerini ${ }^{*}$, Marco Gambarotti ${ }^{2}$, Eric L Staals ${ }^{3}$, Licciana Zanella ${ }^{2}$, Gabriela Sieberova ${ }^{2}$, Alessandra Longhi ${ }^{1}$, \\ Marilena Cesari ${ }^{1}$, Stefano Bonarelli ${ }^{1}$, Piero Picci ${ }^{4}$, Pietro Ruggieri ${ }^{3}$, Marco Alberghini ${ }^{2}$ and Stefano Ferrari ${ }^{1}$
}

\begin{abstract}
Background: Dermatofibrosarcoma protuberans (DFSP) is a relatively common soft-tissue tumor. A more aggressive appearing fibrosarcoma may arise in DFSP, changing its biological behavior. CD34 and apolipoprotein-D are highly expressed in DFSP, but their prognostic significance is uncertain.

Methods: DFSP and fibrosarcomatous-DFSP (FS-DFSP) patients referred to our institute between 1982 and 2009 were identified. Fibrosarcomatous changes, expression of CD34 and apolipoprotein-D were evaluated.

Results: 40 patients, (median age 43 years, 55\% males) were identified. Tumor was located in the limbs in 60\%, in the trunk in 40\%. Thirty-seven patients had localized and 3 had metastatic disease. Thirteen (32\%) patients were FSDFSP. All but one underwent surgery with adequate surgical margins in 72\%. 7 FS-DFSP received also radiotherapy (RT). Chemotherapy was administered to 3 patients with FS-DFSP. With a median follow-up of 49 months, the 5-OS was 90\%. Local recurrence rate was 23\%: 42\% FS-DFSP, 15\% DFSP. Metastases developed in three FS-DFSP patients. The 5-year EFS was 70\% in localized patients. Histology (DFSP 75\% vs. FS-DFSP 52\%, $p=0.002$ ), surgical margins (adequate $74 \%$ vs. inadequate 55\%, $p=0.02$ ), site (limb 47\% vs. trunk 100\%), CD34 expression (CD34 positive: 70\% vs. CD34 negative: $33 \%, p=0.05$ ), and apolipoprotein-D expression (Apo-D positive: 73\% vs. Apo-D negative: 33\%, $p=0.02$ influenced the 5-year EFS, whereas sex, use of RT or number of previous surgical treatments did not.
\end{abstract}

Conclusions: Patients with DFSP have a high survival probability. Site, adequate surgical margins, presence of the fibrosarcomatous component, lack of CD34 expression and apolipoprotein-D influence outcome.

Keywords: dermatofibrosarcoma protuberans, soft tissue sarcoma, Apolipoprotein-D, CD34

\section{Background}

Dermatofibrosarcoma protuberans (DFSP) is a low grade malignant mesenchymal tumor that typically arises in the dermis of the trunk and proximal extremities [1]. DFSP represents 1 to $6 \%$ of all soft tissue sarcomas (STS) $[2,3]$ and its frequency of detection slowly has increased over time [4]. DFSP is characterized by latency of initial diagnosis, slow infiltrative growth and a high rate of local recurrence if not adequately treated. Death due to metastatic disease is very rare $(<5 \%)$ [5]. Histologically, DFSP is usually characterized by uniform spindle

\footnotetext{
* Correspondence: emanuela.palmerini@ior.it

'Chemotherapy, Musculoskeletal Oncology Department, Istituto Ortopedico Rizzoli, via Pupilli 1, Bologna, 40136, Italy

Full list of author information is available at the end of the article
}

shaped cells with elongated neuroid nuclei, proliferating in a storiform growth pattern, infiltrating subcutaneous tissues with a "honeycomb" appearance. In rare cases DFSP shows areas with high-grade fibrosarcomatous changes (more than 5 mitoses/10 HPF, a fascicular growth pattern, increased cellularity and atypia). When dedifferentiated areas represent more than $5 \%$ of tumor tissue, the lesion is classified as fibrosarcomatous ("highgrade") dermatofibrosarcoma protuberans (FS-DFSP) [1]. The prognostic influence of the fibrosarcomatous component of FS-DFSP has been debated [1].

Immunohistochemically, most DFSP stain positively for CD34, whereas, FS-DFSP are CD34 positive in about half of cases [6]. Apolipoprotein-D (Apo-D), a glycoprotein component of human plasma lipid transport system,
C Biomed Central 
has been found to be highly expressed in DFSP by gene arrays [7] and it has been proposed as a novel marker for this neoplasm [8]. The role of Apo-D in DFSP is unknown.

Most DFSPs are cured by wide surgical excision [1]. In some experienced centers, Mohs micrographic surgery (MMS) is routinely used instead of wide excision [5]. Radiotherapy also has been employed [9]. Chemotherapy has been adopted in FS-DFSP patients. Distant metastases generally occur as late sequela following repeated local recurrences. In some of these patients, imatinib has been used successfully $[10,11]$. The target of imatinib in these tumors is the product of a chromosomal translocation that involves chromosomes 17 and 22, resulting in fusion of the collagen type I $\alpha \mathrm{I}$ (COL1A1) and platelet-derived growth factor $\beta(P D G F \beta)$ gene, in both DFSP and FS-DFSP [12]. A FISH probe for $C O L 1 A 1 / P D G F B$ detection is not yet commercially available.

The objective of this study was to retrospectively examine all DFSP and FS-DFSP patients treated at our institution in order to identify tumor- and treatmentrelated factors influencing survival.

\section{Methods}

After obtaining Institutional Review Board approval, all patients diagnosed with DFSP and FS-DFSP between 1982-2009 were identified from the database of the Pathology Department of Musculoskeletal Oncology at the Rizzoli Institute. Two pathologists (M.G. and G.S.) reviewed the slides. Tumors were classified according to the Enzinger \& Weiss criteria: FS-DFSP were identified by the presence of fibrosarcomatous changes (more than 5 mitoses/10 HPF, a "fascicular" growth pattern, increased cellularity and atypia) in at least more than $5 \%$ of tumor tissue [1]. Immunohistochemical expression of CD34 (Qbend-10, 1-100 dilution, Dako, Carpinteria CA, USA) and Apo-D (36C6, 1-200 dilution, Novocastra, New-castle-on-Tyne, UK) were assessed by two pathologists (M-G. and L.Z.) in all patients with adequate tumor tissue. Detection of the two antibodies was performed on a Dako automated immunostainer with universal detection kit streptavidin biotin-alkaline phosphatase/red/detection system Dako after heat (ApoD) and enzyme (CD34) induced antigen retrieval.

All patients surgically treated at our institution with histological diagnosis confirmed were included in the analysis. After 1986, the staging consisted of a computed tomography scan (CT-scan) and/or magnetic resonance imaging of the primary lesion, and a chest CT-scan; other specific tests (bone scan, abdomen CT-scan) were performed only in the case of clinical suspicion. Prior to 1986, a plain chest X-ray and ultrasound of the lesion were performed. Assessment of the surgical margins was based on both the pathology report and the description of the surgical excision. All patients were followed-up with ultrasound, computer tomography or magnetic resonance imaging studies at three to four-months intervals for at least two years, and subsequently at sixmonths intervals for another three years.

Pattern of recurrence for localized patients were defined as follow: local recurrence, when tumor relapse was confined to the primary tumor area; metastases, for distant only metastases: local recurrence plus metastases for local and distant recurrence.

\section{Statistical Analysis}

The following parameters were examined for prognostic value in patients with localized disease: patient sex, tumor anatomic site, surgical margins, histology, CD34 and Apo-D expression, number of previous surgical treatment, use of radiotherapy.

The following categories were compared: tumor site (extremity: at or distal to the shoulder joint and in the groin or leg; trunk: proximal to the shoulder joint and the groin); surgical margins (adequate: wide or radical; inadequate: intralesional, marginal or contaminated margins, according to Enneking's classification)[13]; histology (DFSP vs. FS-DFSP); CD34 and Apo-D expression (positive or negative), number of previous operations $(0$ or $\geq 1$ ); adjuvant treatments (radiotherapy performed within 3 months after tumor excision).

We analyzed overall survival (OS) and event-free survival (EFS). OS time was calculated from the time of admission at our Institute to death or last follow-up visit. EFS time was calculated from the time of admission at our Institute and the occurrence of an event. An event was defined as local recurrence, distant recurrence or death (disease-related or unrelated). All time-to-event end points were modeled using the method of Kaplan and Meier and analyzed by the log-rank test. The results of the Cox model analysis are reported as relative risks (RRs) and 95\% confidence intervals (CIs).

\section{Results}

A total of 51 consecutive patients with histologic diagnosis of DFSP or FS-DFSP made between 1982 and 2009 were identified. Eleven patient were consultation cases, therefore 40 patients were included in the study (Table 1). Reason for admission at our institution was: a new diagnosis in 4 patients (10\%), local recurrence in 19 (47.5\%), scar re-excision 14 (35\%) and distant (lymph node) metastases plus local recurrence in 3 patients (7.5\%). In 13 (32.5\%) patients, sarcomatous changes were documented. In 26 of the 40 evaluable patients tissue samples were available for immunohistochemical assessment of the expression of CD34+ and Apo-D (Figure 1, Table 2). 
Table 1 Distribution of Clinical Pathologic, and Treatment Characteristics

\begin{tabular}{|c|c|}
\hline Number of patients identified & 40 \\
\hline \multicolumn{2}{|l|}{ Sex } \\
\hline Female & $18(45 \%)$ \\
\hline Male & $22(55 \%)$ \\
\hline \multicolumn{2}{|l|}{ Site of primary } \\
\hline Limbs & $24(60 \%)$ \\
\hline Trunk & $16(40 \%)$ \\
\hline \multicolumn{2}{|l|}{ Age } \\
\hline Median (min-max) & 39 yrs (4-80) \\
\hline \multicolumn{2}{|l|}{ Histology } \\
\hline DFSP & $27(67.5 \%)$ \\
\hline FS-DFSP & $13(32.5 \%)$ \\
\hline \multicolumn{2}{|l|}{ Metastases at presentation } \\
\hline None & 37 (92.5\%) \\
\hline Limphonodal & $3(7.5 \%)$ \\
\hline \multicolumn{2}{|l|}{ Previous surgical treatment } \\
\hline Yes & $36(90 \%)$ \\
\hline No & $4(10 \%)$ \\
\hline Median $n^{\circ}$ of operations (min-max) & $1(1-5)$ \\
\hline \multicolumn{2}{|l|}{ Margins * } \\
\hline Wide & $28(72 \%)$ \\
\hline Marginal & $5(13 \%)$ \\
\hline Intralesional & $6(15 \%)$ \\
\hline \multicolumn{2}{|l|}{ Radiotherapy } \\
\hline Yes & $11(27 \%)$ \\
\hline No & $29(73 \%)$ \\
\hline \multicolumn{2}{|l|}{ Chemotherapy } \\
\hline Yes & $3(7.5 \%)$ \\
\hline No & $37(92.5 \%)$ \\
\hline
\end{tabular}

* margins were available in the 39 patients surgically treated

\section{Local Treatment}

Most patients (36/40) had already been surgically treated. Thirty-nine patients $(97.5 \%)$ underwent surgery at our Institute. The amputation rate was 8\% (3/39). One patient refused the planned surgical treatment (amputation) and was lost to follow-up. Adequate surgical margins were detected in 28 of 39 (72\%) surgically treated patients (Table 1). Radiotherapy was administered to 11 patients $* *$ (7 with FS-DFSP).

\section{Chemotherapy}

Adjuvant ifosfamide and epirubicin was administered to 3 patients, all had FS-DFSP. In two cases it was employed after excision of a local recurrence and in one case following an amputation for local recurrence and nodal metastases.

\section{Outcome}

Two patients were lost to follow-up. With a median follow-up of 49 months (9-184), the 5-year overall survival
(OS) was 90\% (95\%CI 80-100) (Figure 2). The 5-year event-free survival (EFS) was 69\% (95\%CI 51-87) in localized patients. Histology (DFSP 75\% (95\%CI 53-97) vs. FS-DFSP $52 \%,(95 \%$ CI $21-83), \mathrm{p}=0.002)$, surgical margins (adequate $75 \%$ (95\% CI 55-95) vs. inadequate $55 \%(95 \%$ CI $16-95), \mathrm{p}=0.02)$, site (extremity $47 \%(95 \%$ CI 22-73) vs. trunk $100 \%, \mathrm{p}=0.005)$, influenced the 5-year EFS, whereas sex, use of RT or number of previous surgical treatments did not (Figure 3). Multivariate analysis (via Cox regression) was performed. Site (extremity RR $36.9,95 \%$ CI 2.1 to $621.7, \mathrm{p}=0.01$ ), sex (female RR $0.08,95 \%$ CI 0.009 to $0.7, p=0.02$ ) and histology (FS-DFSP RR 0.1, 95\%CI 0.01 to $0.8, \mathrm{p}=0.03$ ) independently influenced EFS.

\section{CD34 and apolipoprotein-D expression}

CD34 and Apo-D expression in DFSP and FS-DFSP is shown in Table 2 . The 5 -year EFS was $70 \%(95 \% \mathrm{CI} 43-$ 96) in CD34 positive vs. $33 \%$ (95\%CI 0-71) in CD34 negative patients, $(\mathrm{p}=0.05)$, and $73 \%(95 \% \mathrm{CI} 45-100)$ in Apo-D positive vs. $33 \%$ (95\%CI 0-69) in Apo-D negative patients, $(p=0.02)$ (Figure 4$)$. We did not find significant difference according to histology, site or margins between the two groups. When CD34 and Apo-D were included in the multivariate analysis they lost their prognostic significance.

\section{Pattern of failure in patients with localized disease}

Incidence of local recurrence was 23\% (9/39) overall: $42 \%$ in FS-DFSP and 15\% in DFSP. All relapses occurred in tumors of the limbs, while no recurrences were observed in tumors of the trunk. Three patients with local recurrence developed also pulmonary metastases; all of them presented with FS-DFSP histology; none of the patients with DFSP developed metastases. Local recurrence occurred in 5/28 (18\%) patients with adequate margins and in $4 / 11$ (36\%) patients with inadequate (intralesional or marginal) margins. None of patients with inadequate margins developed distant metastases.

\section{Discussion}

DFSP is a soft tissue malignancy that often shows extensive local invasion but rarely metastasizes [14]. DFSP can lead to significant morbidity from repeated surgical excisions, but infrequently leads to death $[3,5,6,15,16]$.

Dermatofibrosarcoma protuberans with fibrosarcomatous dedifferentiation (FS-DFSP) represents an more aggressive appearing variant of DFSP, in which the prognostic influence of the fibrosarcomatous component is still debated. In our series the high grade variant represented almost a third of the cases, most likely due to a referral center selection bias. 


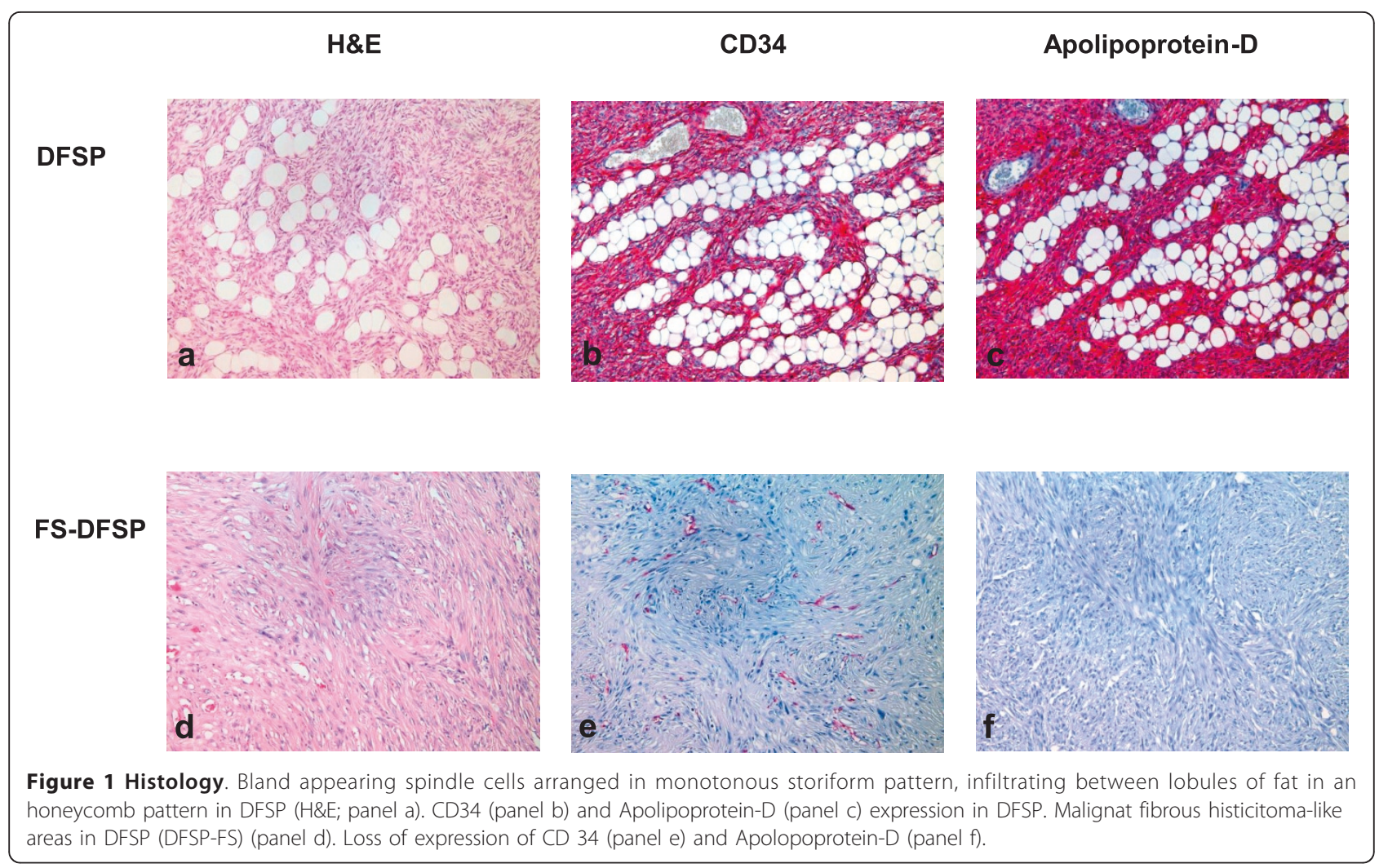

In this study the FS-DFSP patients developed distant recurrence in $23 \%$ and local recurrence in $42 \%$, while DFSP was associated with a $15 \%$ of local recurrence rate and no distant metastases. In other series the rates of local and distant recurrence are similar (Table 3) [6,15-18].

Furthermore the presence of high grade fibrosarcomatous changes in our series is statistically associated with inferior EFS. Therefore, fibrosarcomatous changes in DFSP represent a clinically relevant form of tumor progression. Several authors suggested that fibrosarcomatous

Table 2 Immunoistochemical Expression of CD34 and Apolipoprotein D*

\begin{tabular}{|c|c|c|c|c|}
\hline & $\begin{array}{c}\text { All (26 } \\
\text { pts) } \\
\text { n (\%) }\end{array}$ & $\begin{array}{c}\text { DFSP (15 } \\
\text { pts) } \\
\text { n (\%) }\end{array}$ & $\begin{array}{l}\text { FS-DFSP (11 } \\
\text { pts) } \\
\text { n (\%) }\end{array}$ & \\
\hline \multicolumn{5}{|l|}{ CD34 } \\
\hline Yes & $20(77)$ & $13(87)$ & $7(63)$ & $\begin{array}{l}p= \\
0.3\end{array}$ \\
\hline No & $6(23)$ & $2(13)$ & $4(37)$ & \\
\hline \multicolumn{5}{|c|}{$\begin{array}{l}\text { Apolipoprotein- } \\
\text { D }\end{array}$} \\
\hline Yes & $17(65)$ & $11(73)$ & $6(55)$ & $\begin{array}{l}p= \\
0.4\end{array}$ \\
\hline No & 9 (35) & $4(27)$ & $5(45)$ & \\
\hline
\end{tabular}

* in 26 patients; pts: patients; DFSP: dermatofibrosaroma protuberans; FS-DFSP fibrosarcomatous-DFSP areas within DFSP are associated with higher local recurrence and distant metastases rate [6,18-21]; however, in these studies, the status of surgical margins was not clear. Goldblum studied 18 FS-DFSP patients with adequate margins and reported a relatively low local recurrence rate (22\%) (Table 3) and no distant recurrence. [19]. In our series, 3 out of 13 patients with FS-DFSP and wide margins developed distant relapse. We believe that an higher rate of local and distant recurrence in FS-DFSP compared to DFSP could be related to the presence of high-grade fibrosarcomatous changes and not only to the status of margins. The different results from our and Goldblum studies may be due to differences in patient selection.

Apo-D and CD34 were recently employed as useful markers in differentiating superficial tumors, including DFSP [22]. In our series, the positivity to both membrane markers was documented in about $2 / 3$ of patients. The expression is lower in FS-DFSP compared with DFSP. EFS at 5 years was significantly better in CD34 positive patients. Very similarly, Apo-D positive patients, had an EFS at 5 years of $73 \%$, compared to 33\% in Apo$\mathrm{D}$ negative cases. We did not find significant differences according to histology (DFSP vs. FS-DFSP), site or margins in the 2 groups. The multivariate analysis did not confirm the independent prognostic significance of the expression of Apo-D and CD34, possibly because of the 

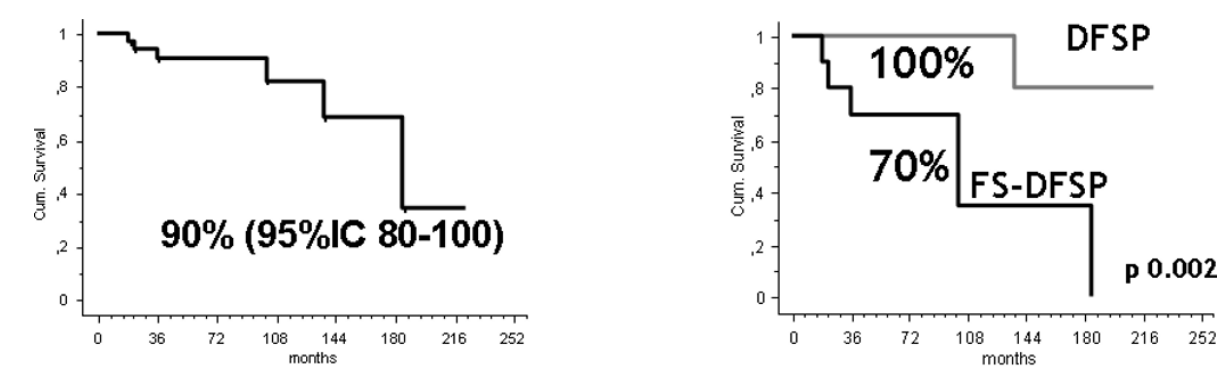

\section{5-year Overall survival (OS)}

Median follow-up of 49 months (9-184)

Figure 2 Overall Survival. Five-year overall survival (OS) in 40 DFSP patients with localized disease and metastatic disease at presentation (left). Five-year overall survival (OS) according to histology (right).

Histology

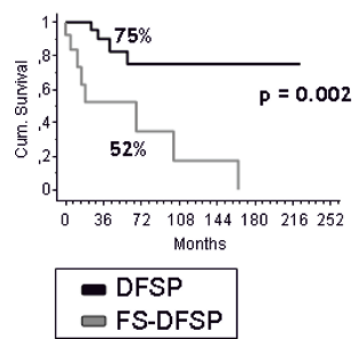

Surgical margins

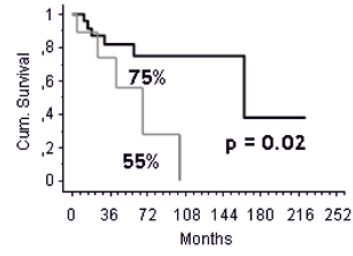

adequate margins $\square$ inadequate margins
Site

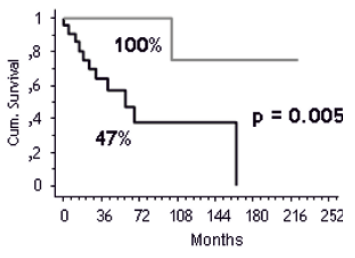

- limbs $\square$ trunk

Figure 3 Five-year event free survival (EFS) according to histology, adequacy of surgical margins and site.
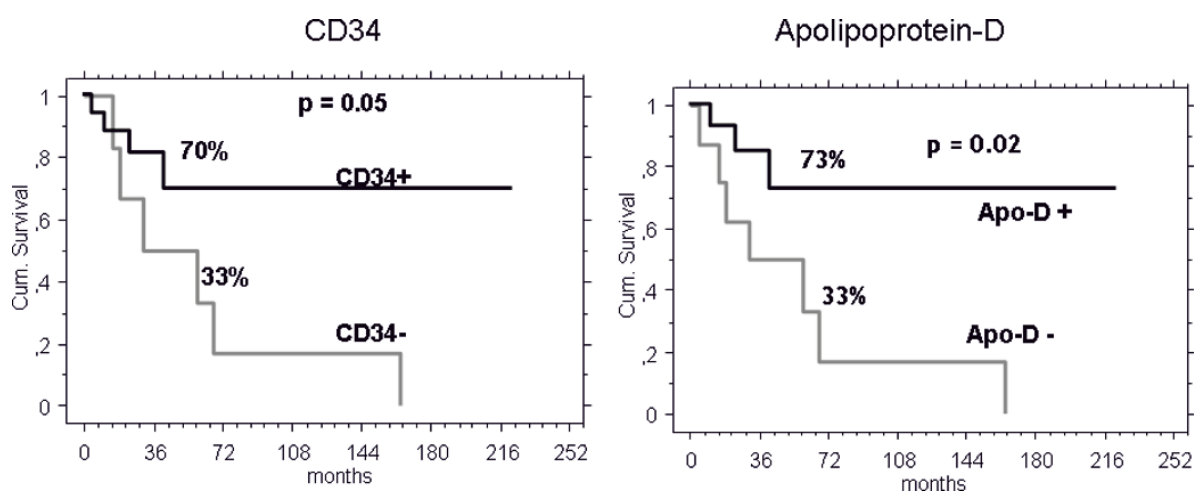

Figure 4 Five-year event free survival (EFS) according to CD34 and Apolipoprotein-D expression.

small sample size. To our knowledge, our series is the first addressing their prognostic role in the subset of DFSP or FS-DFSP. Our observation, an increased risk of local recurrence in patients with lower expression of these markers, should be considered in the planning of the surgical strategy.

\section{Conclusions}

Patients with DFSP have a high survival probability. In this series, limb location, inadequate surgical margins and fibrosarcomatous changes were associated with higher local and metastatic recurrence. The role of adjuvant therapies has yet to be defined. Loss of CD34+ 
Table 3 Local and distant recurrence rate in FS-DFSP

\begin{tabular}{lccc}
\hline Ref. & Pts $^{\circ}$ & Distant & Local \\
\hline Palmerini et al., current study & 13 & $23 \%$ & $42 \%$ \\
Mentzel T et al., AJSP 1998 [6] & 41 & $15 \%$ & $58 \%$ \\
Bowne WB et al., Cancer 2000 [15] & 25 & $8 \%$ & $52 \%$ \\
Fiore M et al, JCO 2005 [16] & 7 & $28 \%$ & $28 \%$ \\
Abbott JJ et al. AJSP 2006 [17] & 41 & $10 \%$ & $20 \%$ \\
Goldblum JR et al., AJSP 2000 [18] & 18 & $0 \%$ & $22 \%$ \\
\hline
\end{tabular}

and/or Apolipoprotein D expression are associated with a worse prognosis in this series.

\section{Author details}

${ }^{1}$ Chemotherapy, Musculoskeletal Oncology Department, Istituto Ortopedico Rizzoli, via Pupilli 1, Bologna, 40136, Italy. ${ }^{2}$ Surgical Pathology,

Musculoskeletal Oncology Department, Istituto Ortopedico Rizzoli, via Pupill 1, Bologna, 40136, Italy. ${ }^{3}$ Orthopaedic Surgery, Musculoskeletal Oncology Department, Istituto Ortopedico Rizzoli, via Pupilli 1, Bologna, 40136, Italy. ${ }^{4}$ Laboratory of Oncologic Research, Musculoskeletal Oncology Department, Istituto Ortopedico Rizzoli, via Pupilli 1, Bologna, 40136, Italy.

\section{Authors' contributions}

The work presented here was carried out in collaboration between all authors. PE, GM and SLE defined the research theme; LZ, GM, SG and MA: carried out the pathological studies and Immunohistochemical assays; LA, CM and SB co-worked on associated data collection and their interpretation; $E P, P P, P R, A M$, and FS discussed analyses, interpretation, and drafted the manuscript. All authors read and approved the final manuscript.

\section{Competing interests}

The authors declare that they have no competing interests.

Received: 5 November 2011 Accepted: 27 January 2012 Published: 27 January 2012

\section{References}

1. Weiss SW, Goldblum JR: Fibrohistiocytic tumors of intermediate malignancy. Enzinger \& Weiss's Soft Tissue Tumors. 5 edition. Missouri: Mosby Elsevier; 2008, 371-383.

2. Kransdorf MJ: Malignant soft-tissue tumors in a large referral population: distribution of diagnoses by age, sex, and location. AJR Am J Roentgenol 1995, 164:129-134.

3. Chang CK, Jacobs IA, Salti GI: Outcomes of surgery for dermatofibrosarcoma protuberans. Eur J Surg Oncol 2004, 30:341-345.

4. Laskin WB: Dermatofibrosarcoma protuberans. CA Cancer J Clin 1992, 42:116-125.

5. Gloster HM Jr: Dermatofibrosarcoma protuberans. J Am Acad Dermatol 1996, 35:355-374.

6. Mentzel T, Beham A, Katenkamp D, Dei Tos AP, Fletcher CD: Fibrosarcomatous ("high-grade") dermatofibrosarcoma protuberans: clinicopathologic and immunohistochemical study of a series of 41 cases with emphasis on prognostic significance. Am J Surg Pathol 1998, 22:576-587.

7. Linn SC, West RB, Pollack JR, Zhu S, Hernandez-Boussard T, Nielsen TO, Rubin BP, Patel R, Goldblum JR, Siegmund D, Botstein D, Brown PO, Gilks $C B$, van de Rijn M: Gene expression patterns and gene copy number changes in dermatofibrosarcoma protuberans. Am J Pathol 2003, 163:2383-2395.

8. West RB, Harvell J, Linn SC, Liu CL, Prapong W, Hernandez-Boussard T, Montgomery K, Nielsen TO, Rubin BP, Patel R, Goldblum JR, Brown PO, van de Rijn M: Apo $D$ in soft tissue tumors: a novel marker for dermatofibrosarcoma protuberans. Am J Surg Pathol 2004, 28:1063-1069.

9. Suit HD, Spiro IJ, Spear M: Benign and low-grade tumors of the soft tissues: role for radiation therapy. Cancer Treat Res 1997, 91:95-105.
10. Maki RG, Awan RA, Dixon RH, Jhanwar S, Antonescu CR: Differential sensitivity to imatinib of 2 patients with metastatic sarcoma arising from dermatofibrosarcoma protuberans. Int J Cancer 2002, 100:623-626.

11. Rubin BP, Schuetze SM, Eary JF, Norwood TH, Mirza S, Conrad EU, Bruckner JD: Molecular targeting of platelet-derived growth factor B by imatinib mesylate in a patient with metastatic dermatofibrosarcoma protuberans. J Clin Oncol 2002, 20:3586-3591.

12. Llombart B, Sanmartín O, López-Guerrero JA, Monteagudo C, Serra C, Requena C, Poveda A, Vistós JL, Almenar S, Llombart-Bosch A, Guillén C: Dermatofibrosarcoma protuberans: clinical, pathological, and genetic (COL1A1-PDGFB) study with therapeutic implications. Histopathology 2009, 54:860-872.

13. Enneking WF, Spanier SS, Goodman MA: Current concepts review. The surgical staging of musculoskeletal sarcoma. J Bone Joint Surg 1980, 62A:1027-1030.

14. Koh CK, Ko CB, Bury HP, Wyatt EH: Dermatofibrosarcoma protuberans. Int J Dermatol 1995, 34:256-260.

15. Bowne WB, Antonescu CR, Leung DH, Katz SC, Hawkins WG, Woodruff JM, Brennan MF, Lewis JJ: Dermatofibrosarcoma protuberans: A clinicopathologic analysis of patients treated and followed at a single institution. Cancer 2000, 88:2711-2720.

16. Fiore M, Miceli R, Mussi C, Lo Vullo S, Mariani L, Lozza L, Collini P, Olmi P, Casali PG, Gronchi A: Dermatofibrosarcoma protuberans treated at a single institution: a surgical disease with a high cure rate. $J$ Clin Oncol 2005, 23:7669-7675.

17. Abbott JJ, Oliveira AM, Nascimento AG: The prognostic significance of fibrosarcomatous transformation in dermatofibrosarcoma protuberans. Am J Surg Pathol 2006, 30:436-443.

18. Goldblum JR, Reith JD, Weiss SW: Sarcomas arising in dermatofibrosarcoma protuberans: a reappraisal of biologic behavior in eighteen cases treated by wide local excision with extended clinical follow up. Am J Surg Pathol 2000, 24:1125-1130.

19. Ding J, Hashimoto H, Enjoji M: Dermatofibrosarcoma protuberans with fibrosarcomatous areas. A clinicopathologic study of nine cases and a comparison with allied tumors. Cancer 1989, 64:721-729.

20. Connelly JH, Evans HL: Dermatofibrosarcoma protuberans. A clinicopathologic review with emphasis on fibrosarcomatous areas. Am J Surg Pathol 1992, 16:921-925.

21. Pizzarro JB, Fanburg JC, Mietinem M: Dermatofibrosarcoma protuberans (DFSP) with fibrosarcomatous transformation: re-explored. Modern Pathol 1997, 10:13A.

22. Lisovsky M, Hoang MP, Dresser KA, Kapur P, Bhawan J, Mahalingam M: Apolipoprotein D in CD34-positive and CD34-negative cutaneous neoplasms: a useful marker in differentiating superficial acral fibromyxoma from dermatofibrosarcoma protuberans. Mod Pathol 2008 , 21:31-38.

doi:10.1186/2045-3329-2-4

Cite this article as: Palmerini et al.: Fibrosarcomatous changes and expression of CD34+ and apolipoprotein-D in dermatofibrosarcoma protuberans. Clinical Sarcoma Research 2012 2:4.

\section{Submit your next manuscript to BioMed Central and take full advantage of:}

- Convenient online submission

- Thorough peer review

- No space constraints or color figure charges

- Immediate publication on acceptance

- Inclusion in PubMed, CAS, Scopus and Google Scholar

- Research which is freely available for redistribution

Submit your manuscript at www.biomedcentral.com/submit
Ciomed Central 